\title{
Central motor pathways in patients with mirror
}

\section{movements}

\author{
T C Britton, B-U Meyer, R Benecke
}

\begin{abstract}
Central motor pathways were investigated in three patients with congenital mirror movements using magnetic motor cortex stimulation. Response thresholds, amplitudes and latencies were normal. The projection of the corticomotoneuronal pathways was assessed by placing the coil over the vertex and comparing the size of responses in the first dorsal interosseous (FDI) muscles evoked by clockwise and anticlockwise coil currents. In normal subjects, right FDI responses are larger with clockwise currents than with anticlockwise currents at the same stimulation strength and vice versa. In two out of three patients with congenital mirror movements, this sensitivity of response amplitude to coil current direction was reversed. The third patient with congenital mirror movements and a fourth patient with acquired mirror movements had responses which were normally sensitive to current direction. These findings support the hypothesis that some cases of congenital mirror movements may be due to abnormal projection of corticomotoneuronal pathways.
\end{abstract}

Mirror movements are a special type of associated movement in which voluntary movements performed by one part of the body, particularly the arm or hand, are involuntarily and symmetrically performed by the other side as a result of bilateral activation of homologous muscles. ${ }^{12}$ Mirror movements occur in children during development but normally disappear with maturation. Persistence of mirror movements into adult life, termed congenital mirror movements, is considered pathological and may be associated with other developmental abnormalities including the Klippel-Feil syndrome $\mathrm{e}^{34}$ and Kallmann's syndrome. ${ }^{5}$ Mirror movements can also appear with a variety of acquired neurological conditions. ${ }^{12}$

Anatomically, congenital mirror movements are often associated with midline fusion disorders affecting the motor pathways. Incomplete pyramidal tract decussation has been reported in the necropsy examinations of two cases that had congenital mirror movements associated with the Klippel-Feil syndrome. ${ }^{34}$ Dysraphic defects at different levels within the central nervous system are thought to underlie congenital mirror movements associated with the syndrome of anosmia and hypophyseal dysfunction. ${ }^{5}$

Neurophysiological evidence for abnormal ipsilateral corticomotoneuronal projections has recently been reported in brief abstract form in two patients with congenital mirror movements ${ }^{6}$ and in one patient with mirror movements associated with Klippel-Feil syndrome $^{7}$ using the technique of trans-cranial electrical motor cortex stimulation. This study on three patients with congenital mirrror movements reports that abnormal corticomotoneuronal projections can be demonstrated with the technique of magnetic motor cortex stimulation ${ }^{8}$ in two of the three cases. However, the third patient with congenital mirror movements and a patient with acquired mirror movements as a result of internal capsular infarction had normally projecting corticomotoneuronal pathways. These findings have implications for the pathophysiological significance of nondecussated pyramidal pathways in congenital mirror movements.

\section{Methods}

PATIENTS AND SUBJECTS

Four patients with mirror movements were investigated. Three had had mirror movements since childhood. The fourth developed mirror movements one week following a left internal capsular infarction.

Case 1 A 52 year old right handed man had noticed mirror movements of his hands since childhood. He was moderately disabled in fine motor tasks of the hands. Pregnancy and early development were otherwise normal. On examination there were non-suppressible mirror movements on finger or wrist movements of either side. Simultaneous symmetrical flexion-extension movements of the wrists could be made normally, but the patient experienced considerable difficulty in producing simultaneous flexion of one wrist and extension of the other. Sequential opposition of the thumb to each of the fingers was slow. No mirror movements were observed in the legs. Muscle strength, tone and reflexes in the upper and lower limbs were normal. The neck was of normal length and a cervical spine radiograph was normal. Sense of smell was normal.

Case 2 A 26 year old man, with a mild right sided hemiplegia since birth, had noticed involuntary movements of his left hand when 
moving with his right hand and, additionally, involuntary movements of his right hand when attempting to move only his left hand. On examination he had a right hemiparetic gait and a mild right hemiparesis with increased muscle tone and brisk reflexes on the right. Mirror movements of the left hand were observed when he attempted to move only the right hand and mirror movements of the right hand were seen when he attempted to move only the left hand. CT of the head showed hemi-atrophy of the left cerebral cortex. There was no abnormality of the neck and sense of smell was normal.

Case 3 A 38 year old right handed woman had been aware of mirror movements in her hands all her life. She was the second of identical twins. Pregnancy and development were otherwise normal. On examination there were nonsuppressible mirror movements of both hands, which were present with self initiated movements as well as with externally triggered (reaction time paradigm) movements. Fine finger movements were mildly impaired. She had considerable difficulty in producing alternating flexion-extension movements of one wrist and extension-flexion movements of the other. Muscle strength, tone and tendon reflexes were normal. There was no abnormality of the neck and sense of smell was normal.

Case 4 A 59 year old right handed hypertensive man suddenly developed a severe right hemiparesis. CT revealed a hyperdense lesion in the region of the left internal capsule compatible with cerebral haemorrhage. Three weeks later, after considerable functional recovery, he noticed that attempts to move his right hand resulted in associated mirror movements in the left hand; movements of his left hand, however, were not accompanied by movements of the right.

A fifth patient, aged 48 , with a left hemiplegia as a result of embolic occlusion of the right middle cerebral artery was also studied.

Five male normal subjects (age range 27 to 31 years, mean 28 years) were studied. Ethical committee approval for these investigations had been obtained.

\section{RECORDING AND ANALYSIS}

Electromyographic (EMG) recordings were taken from both first dorsal interosseous (FDI) musc̀les, using $\mathrm{Ag} / \mathrm{AgCl}$ surface electrodes placed over the muscles. Signals were amplified by a Tönnies Myograph II (Tönnies Medizinische Elecktronik, Freiburg, Germany) with bandpass filtering between $20 \mathrm{~Hz}$ and $3000 \mathrm{~Hz}$. Data was then collected and stored on floppy disks using a Tandon personal computer (IBM PC compatible) and AUTOLAB data collection programmes (sampling frequency $8000 \mathrm{~Hz} /$ channel; AUTOLAB, Kunze Software, Düsseldorf, Germany). Onset latencies of responses were measured from the first deviation from the baseline by visual inspection of the signals on the computer screen. Response amplitudes (peak to peak) were measured by computer.

The motor cortex was stimulated using the commercially available MAGSTIM 200 (Novametrix Medical Systems, Connecticut, USA). The coil (consisting of 19 turns of copper wire; inner diameter $5.5 \mathrm{~cm}$, outer diameter $12 \mathrm{~cm}$ ) was laid flat against the scalp and was centred carefully over the vertex.

It has previously been noted that, with this make of stimulator (not necessarily with others ${ }^{9}$ ) and with the coil centred over the vertex, currents flowing clockwise around the coil (as viewed from above) normally produce larger responses in the right FDI muscle than the left FDI, whereas anticlockwise currents evoke larger responses in the left FDI than right FDI. ${ }^{10}$ This was confirmed in our normal subjects. Given the known contralateral emphasis (particularly for hand muscles) of the corticomotoneuronal projection, the most likely explanation for this result is that clockwise currents stimulate the left cerebral hemisphere more effectively (at least in terms of evoking hand muscle responses) than the right cerebral hemisphere and that anticlockwise coil currents stimulate the right cerebral hemisphere more than the left.

Thus one method of studying the corticomotoneuronal projections in normal subjects and in patients with mirror movements would be to compare directly the size of responses evoked in the two FDI muscles with clockwise currents (when, in normal subjects, right FDI responses are larger than left FDI responses) or with anticlockwise currents (when, in normal subjects, left FDI responses are larger than right FDI responses). The occurrence of reversed responses (for example, clockwise currents producing larger responses in the left FDI than the right FDI, or vice versa) could then be interpreted as indicating a pathological, ipsilaterally projecting corticomotoneuronal tract. There is, however, a problem with such reasoning in that the size of an individual FDI response may be significantly influenced not only by the relative proportion of contra- and ipsilaterally projecting corticomotoneuronal axons but also by other factors (electrode placement, peripheral nerve lesions, lesions of central motor pathways such as lacunar infarction).

To avoid these confounding factors, the size of responses evoked by clockwise coil currents was compared with the size of responses evoked by anticlockwise currents at the same stimulation strength and in the same FDI muscle. In normal subjects, right FDI responses are larger with clockwise coil currents than anticlockwise coil currents at the same stimulation strength (within the range of stimulation strengths from threshold to 1.5 times threshold): for left FDI muscles, anticlockwise currents produce larger responses than clockwise currents. The implications of these normal findings with the corticomotoneuronal projections and the interpretation of reversed response patterns are raised in the discussion.

With the coil position carefully centred over the vertex, responses were recorded in FDI 
L FDI
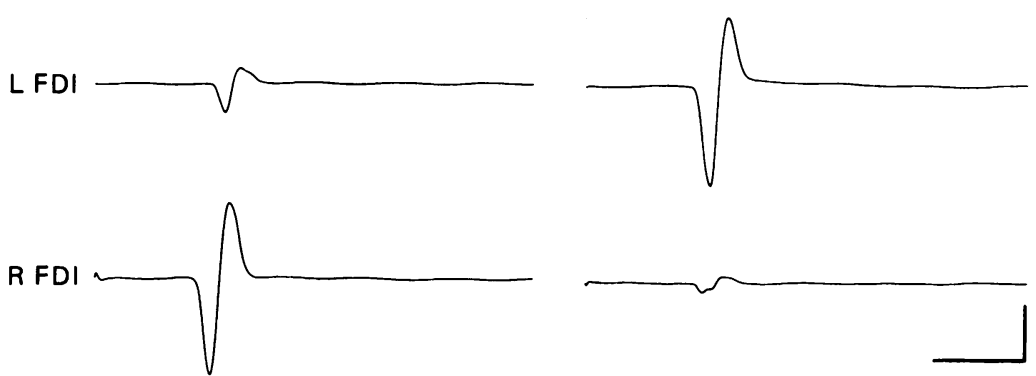

Figure 1 Electromyographic recordings from the left (upper traces) and right (lower traces) first dorsal interosseous (FDI) muscles in a normal subject following magnetic brain stimulation with clockwise coil currents (left side of figure) and anticlockwise coil currents (right side of figure). The coil was centred over the vertex and the stimulation strength was $75 \%$ of the maximum stimulator output. Note how in the left FDI a larger response is produced by an anticlockwise coil current, while in the right FDI a larger response is produced by a clockwise coil current. Calibration bars $20 \mathrm{~ms}$ and $1 \mathrm{mV}$.

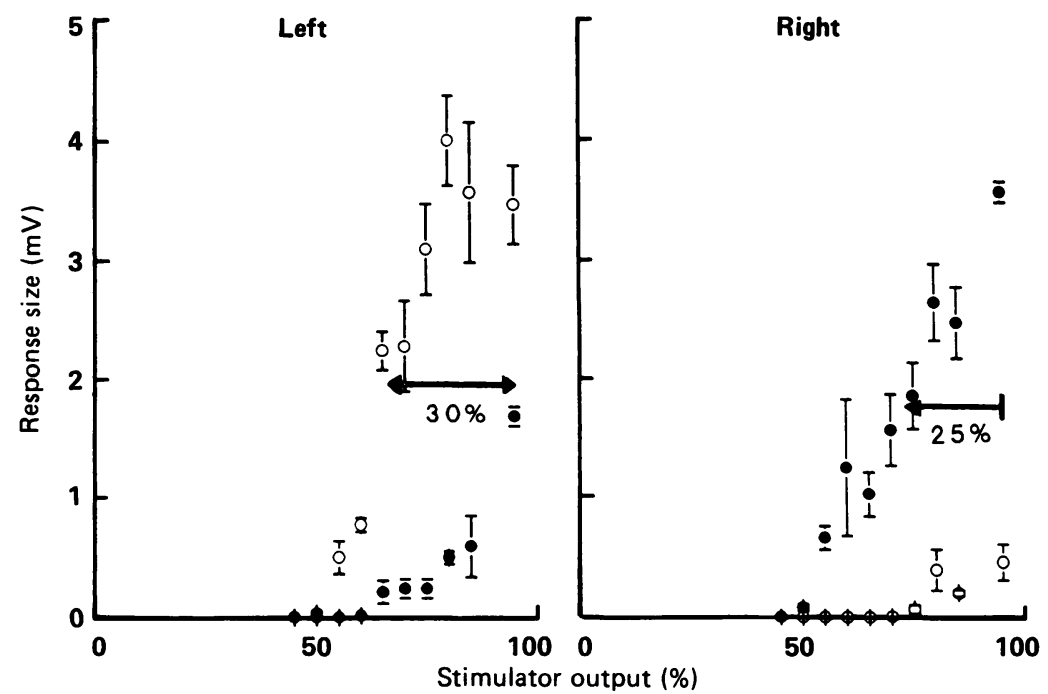

Figure 2 Graphs of average peak to peak size of responses elicited in the left (left half of figure) and right (right half of figure) first dorsal interosseous (FDI) muscles of a normal subject following magnetic brain stimulation with clockwise (filled circles) and anticlockwise (open circles) coil currents plotted against stimulation strength expressed as percent of maximal output of the stimulator. Note how clockwise coil currents produce larger responses in the right FDI than anticlockwise currents at the same stimulation strength and vice versa. This difference in the size of responses with currents of different orientation has been quantified by subtracting the stimulation strength required to produce a half maximal response with anticlockwise currents from that required with clockwise currents.
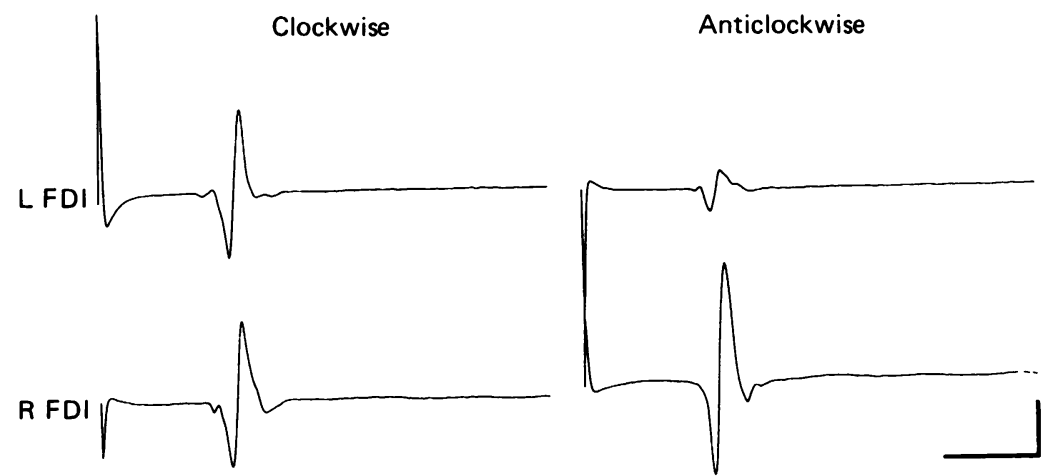

Figure 3 Electromyographic recordings from the left (upper traces) and right (lower traces) first dorsal interosseous (FDI) muscles in a patient with congenital mirror movements (case 1) following magnetic brain stimulation with clockwise coil currents (left side of figure) and anticlockwise coil currents (right side of figure). The coil was centred over the vertex and the stimulation strength was $90 \%$ of the maximum stimulator output. Note how in the left FDI a larger response is produced by a clockwise coil current, while in the right FDI a larger response is produced by an anticlockwise coil current. This is the opposite pattern from normal (see fig 1). Calibration bars $20 \mathrm{~ms}$ and $0.5 \mathrm{mV}$. muscles with clockwise and anticlockwise currents (direction of coil current was changed by simply turning over the coil) using a series of stimulation strengths between 30 and $100 \%$ of maximum output of the stimulator. At least five responses were obtained for each stimulation strength. During stimulation, subjects were asked to relax completely and this was checked by constant display of the surface EMG on an oscilloscope.

To quantify the difference in response size with different coil currents, the mean peak to pleak amplitude of the evoked responses was calculated for each stimulation strength and then plotted on a graph (fig 2). A line was drawn across the graph at a height equal to half the size of the largest response obtained at rest. The stimulation strength (expressed as percent of maximal stimulator output) needed to produce such a half maximal response when the current flowed anticlockwise around the coil was subtracted from the stimulation strength needed when the current flowed clockwise. If response of half maximal size were not evoked when the coil current flowed in the non-preferential direction, then the highest stimulation strength that was used in the particular subject was employed in this calculation. For right FDI muscles of normal subjects this results in a negative value, and for left FDI muscles a positive value.

For the purposes of obtaining cortically evoked responses with the shortest latency, magnetic stimulation of the motor cortex was repeated with pre-activation of the muscle and with the stimulator output set at 1.5 times threshold for responses in the relaxed muscle. ${ }^{112}$ Peak to peak size of responses evoked with pre-activation of the muscle were also measured.

\section{Results}

Normal subjects

Responses in relaxed FDI muscles could be obtained in all normal subjects with the coil current in either direction. Threshold for responses in right FDI muscles (mean $46 \%$ of maximum stimulator output; range $40 \%-60 \%$ ) was always lower with clockwise coil currents (when viewed from above) than with anticlockwise currents, whereas threshold for responses in left FDI muscles (mean $48 \%$; range 40 $65 \%$ ) was always lower with anticlockwise currents. Amplitude of the responses in an FDI muscle was dependent on stimulation strength and current direction (figs 1,2). For the same stimulation strength, the peak to peak amplitude of responses in right FDI muscles were always greater with clockwise coil currents than with anticlockwise currents. The opposite was true for the left FDI muscles. To produce a response of half maximal size in the right FDI, the stimulation strength required with clockwise coil currents was $22 \%$ (range $18 \%$ to $30 \%$ ) less than that for anticlockwise currents, while for half maximal responses in the left FDI the stimulation strength required with clockwise currents was $18 \%$ (range $10 \%$ to $32 \%$ ) more than that for anticlockwise currents. 


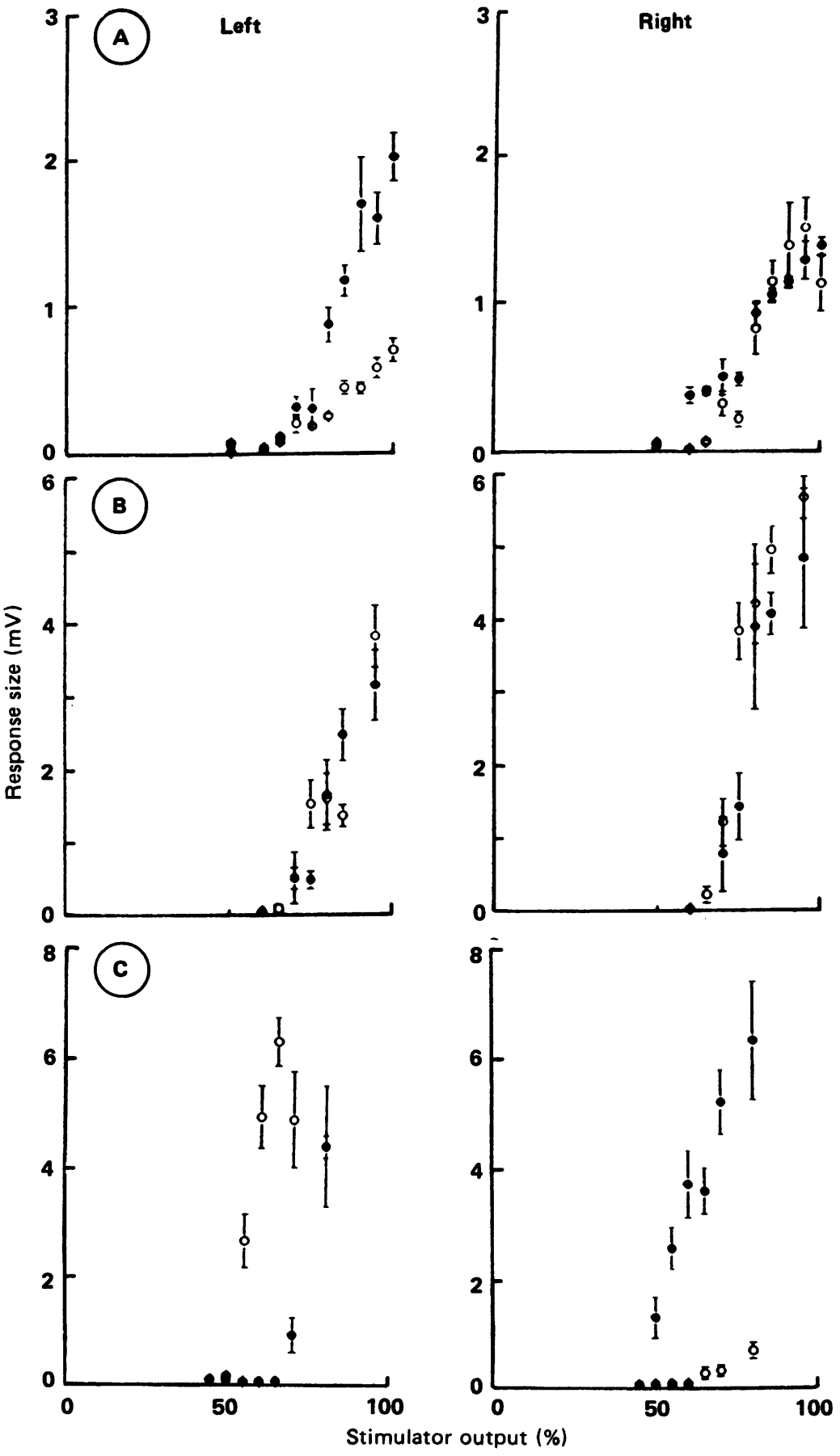

Figure 4 Graphs of average peak to peak size of responses elicited in the left (left half of figure) and right (right half of figure) first dorsal interosseous (FDI) muscles of three patients with congenital mirror movements, following magnetic brain stimulation with clockwise (filled circles) and anticlockwise (open circles) coil currents plotted against stimulation strength expressed as percent of maximal output of the stimulator. Note how in $A$ (case 1) and $B$ (case 2) responses in the right FDI were larger or of equal size when the coil current was anticlockwise compared to when the coil current was clockwise: this is the opposite of the normal findings. Conversely responses in the left FDI were larger or of equal size when the coil current was clockwise compared to when the coil current was anticlockwise, which is again an abnormal finding. These results suggest that the pyramidal pathways in these two patients are not normally decussated. However, $a$ third patient with congenital mirror movements ( $C$; case 3$)$ had responses which were dependent on coil current direction in a normal fashion.

With pre-activation of the muscle, stimulation strength at 1.5 times threshold and use of preferential coil current direction, mean response latencies were $21 \cdot 2 \mathrm{~ms}$ (range $18 \cdot 8$ to $22 \cdot 4$ $\mathrm{ms}$ ) for the right FDI and $21 \cdot 3 \mathrm{~ms}$ (range $20 \cdot 7$ to $23.0 \mathrm{~ms}$ ) for the left FDI.
Patients with congenital mirror movements

Responses could be obtained in both FDI muscles in each patient following magnetic brain stimulation. Threshold for responses (range 45-60\%) lay within the normal range, but in three out of six sides the threshold for clockwise and anticlockwise coil currents was equal, in contrast to the normal findings (fig 3).

The amplitude of responses is plotted against stimulation strength in figure 4 . The graphs for case 1 and case 2 show qualitative and quantitative differences from the findings in normal subjects. Qualitatively, the size of responses in an FDI muscle was not dependent on coil current direction in the same way that it was for normals. Thus for right FDI muscles anticlockwise coil currents produced equally sized or larger responses than clockwise currents at the same stimulation strength (which is the opposite of that found in normals). Furthermore, in these two patients, left FDI responses were preferentially evoked by clockwise currents (in normal subjects anticlockwise currents produced larger responses). Quantitatively, in order to produce responses of half maximal size in the right FDI muscles, the stimulation strength required with clockwise coil currents was $0 \%$ (case 1 ) and 3\% more (case 2; mean for normal subjects $22 \%$ less) than that for anticlockwise currents, while for half maximal responses in the left FDI the stimulation strength required with clockwise currents was $16 \%$ less (case 1 ) and $0 \%$ (case 2; mean for normals $18 \%$ more) than that for anticlockwise currents.

In cases 1 and 2 , we made the additional observation that the size of responses in the right FDI muscles could be increased by moving the coil over the right (ipsilateral) cerebral hemisphere, while the size of left FDI responses could be increased by moving the coil towards the left cerebral hemisphere.

In case 3 , response size varied with coil current direction in a normal manner. That is, clockwise currents evoked larger responses in the right FDI than anticlockwise currents at the same stimulation strength and vice versa.

With pre-activation of the muscle, stimulation strength at 1.5 times threshold and use of preferential coil current direction, cortically evoked response latencies and amplitudes in all three patients with congenital mirror movements were found to be within our normal range. Response configurations also appeared normal although this was not studied systematically.

\section{Patient with acquired mirror movements}

Responses could be obtained in the relaxed FDI muscles of both sides, although the responses on the hemiparetic side were much smaller than those on the non-paretic side. Threshold for responses were within our normal range (right $65 \%$; left $50 \%$ ). Amplitudes of responses were dependent on stimulation strength and current direction in a normal manner (fig 5). Responses in the right FDI muscle were larger with clockwise coil currents than with anticlockwise currents for the same stimulation strength. With muscle pre-activa- 


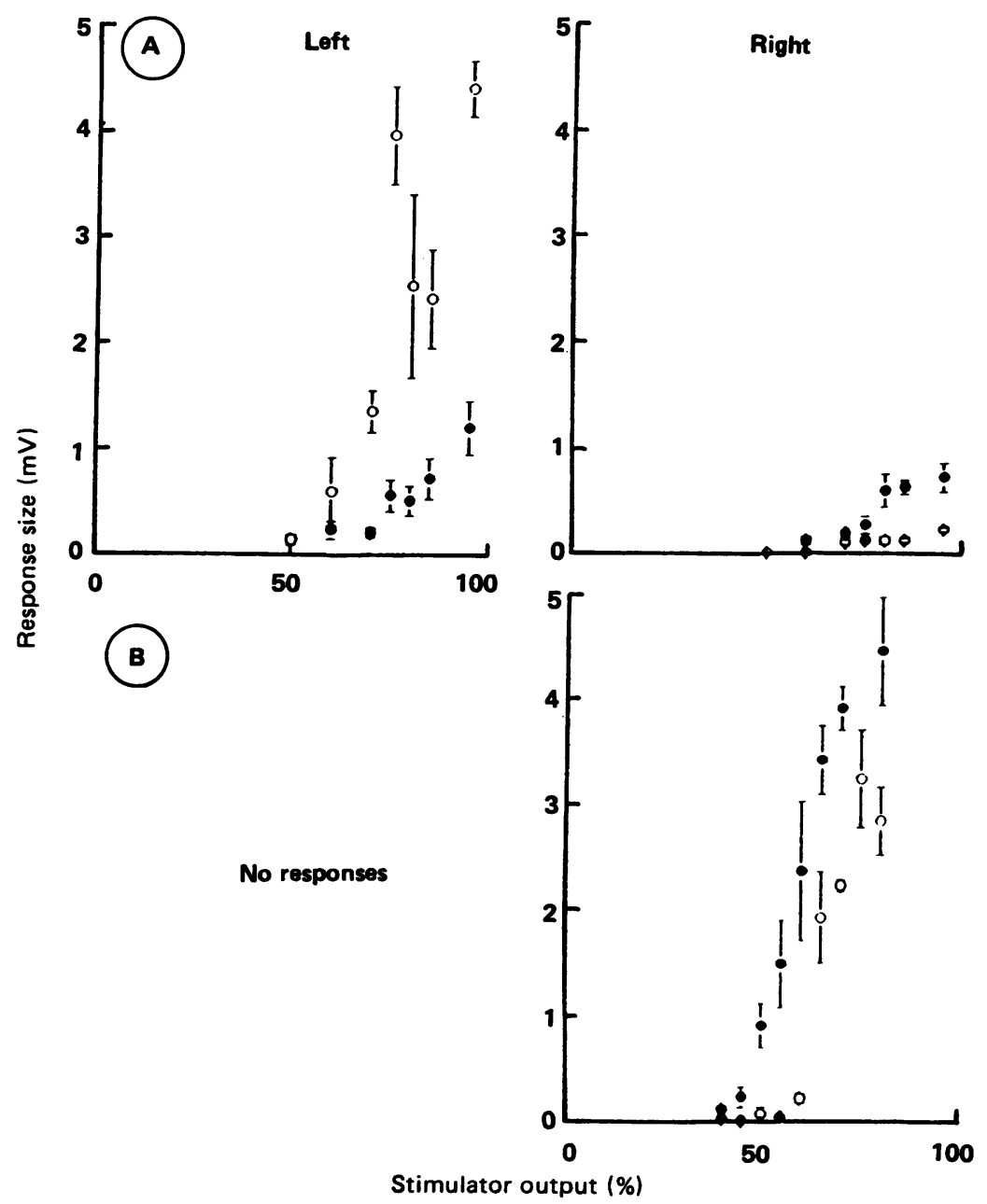

Figure 5 Graphs of average peak to peak size of cortically evoked responses in the left (left half of figure) and right (right half of figure) first dorsal interosseous (FDI) muscles of one patient with acquired mirror movements (upper graphs) and of one patient with a complete right hemiplegia (lower graphs) elicited with clockwise (filled circles) and anticlockwise (open circles) coil currents plotted against stimulation strength expressed as percent of maximal output of the stimulator. Note how the responses are dependent on coil current direction in a normal manner: that is clockwise coil currents produced larger responses than anticlockwise currents at the same stimulation strength in the right FDI, while anticlockwise currents were better at stimulating the left FDI. No responses could be obtained in the completely paralysed right FDI.

tion, cortically evoked response latencies were within normal limits, although somewhat slower in the right FDI $(22.4 \mathrm{~ms})$ compared with the left $(21 \cdot 7 \mathrm{~ms})$. Response amplitude was also reduced on the right $(1.6 \mathrm{mV}$ compared to $6.7 \mathrm{mV}$ ).

\section{Patient with right $C V A$}

Responses following magnetic brain stimulation could be obtained in the right FDI muscle with both clockwise and anticlockwise coil currents, but no responses could be evoked in the left FDI. Responses in the right FDI were normal for threshold $(45 \%)$ and for the direction of current that produced the larger responses for a given stimulation strength. The strength of stimulation required to produce a half maximal response was $60 \%$ with a clockwise coil current and $70 \%$ with an anticlockwise current. The presence of responses in the right FDI muscle with both clockwise and anticlockwise coil currents in this patient indicates that bilaterally projecting corticomotoneuronal pathways are not necessary to explain the finding of bilateral responses in normal subjects when the coil is centred over the vertex.

\section{Discussion}

We have demonstrated that hand muscle responses evoked by magnetic motor cortex stimulation in two out of three patients with congenital mirror movements were preferentially elicited by using the opposite direction of coil current to that which produces the largest responses in normal subjects. This suggests that either: 1) their primary motor cortices were different from normal and were more sensitive to stimulation with coil currents in the opposite direction to normal (that is, clockwise coil currents stimulated the right cerebral hemisphere more effectively than the left hemisphere) or 2) the corticomotoneuronal component of the pyramidal pathways projected to spinal alpha motor neurons abnormally. We favour the latter hypothesis in view of our observation that moving the coil over the ipsilateral hemisphere in these cases often further increased the size of the response. In addition, given that the threshold, latency and size of responses evoked by magnetic stimulation were normal, a major abnormality in the architecture of the motor cortices seems unlikely. Furthermore, such a hypothesis would be in keeping with the results of electrical motor cortex stimulation. ${ }^{67}$

This study has also shown that congenital mirror movements are not invariably associated with abnormal corticomotoneuronal projections. The third patient, whose congenital mirror movements were clinically indistinguishable from the mirror movements of the first and second patients, had entirely normal motor responses to magnetic stimulation, including responses which were appropriately sensitive to the direction of current. The fast corticomotoneuronal component of the pyramidal tracts therefore projected normally to spinal alpha motor neurons, although this does not exclude the possibility that the projection of slower components of the pyramidal pathway might have been abnormal.

What can be said of the pathophysiological basis of congenital mirror movements? The appearance of mirror movements on attempting to move only one side of the body depends upon the original command from high centres within the brain to move only one side of the body inappropriately reaching the spinal alpha motor neurons of both sides. Clearly, several different routes, via pyramidal or extrapyramidal pathways, could be suggested to account for this abnormal transmission..$^{13}$ The initial command might be incorrectly sent to motor centres on both sides of the brain (for example, to the motor cortices of both cerebral hemispheres), or signals in the descending motor pathways from motor centres of one side of the brain (for example, pyramidal pathways) might activate spinal structures bilaterally as a result of the abnormal functional/anatomical projection of such pathways, or activity on one side of the spinal cord might cross to the other 
side as a result of abnormal interneuronal connections.

Could the abnormal ipsilateral corticomotoneuronal projections as revealed in two out of our three patients explain the appearance of congenital mirror movements? In normal subjects making unilateral hand movements only the contralateral motor cortex is activated. ${ }^{14}$ 15 A totally non-decussated (ipsilaterally projecting) corticomotoneuronal projection, by itself, could not therefore account for mirror movements. Assuming that motor cortex activation occurs normally, what is required is that activity in the motor neurons of one cerebral motor cortex reaches the alpha motor neurons on both sides of the spinal cord. This could be effected either by individual pyramidal axons branching to synapse directly with alpha motor neurons on both sides of the spinal cord or by a proportion of activated cortical motor neurons projecting ipsilaterally and a proportion projecting contralaterally. Farmer et $a l,{ }^{7}$ on the basis of a narrow peak on cross-correlograms between motor units in the hand muscles of a patient with Klippel-Feil syndrome, showed that the spinal motor neurons subserving homologous hand muscles on each side receive synaptic input from a common neuron and they suggested that the common neuron was a cortical motor neuron with an abnormally branched axon.

The finding that one of our patients with congenital mirror movements had normal corticomotoneuronal pathways as assessed by magnetic motor cortex stimulation is important since it implies either that there is more than one pathophysiological cause of congenital mirror movements or that the abnormal corticomotoneuronal projections seen in the other patients is not the pathological cause of the mirror movements, merely an associated feature. That mirror movements are also seen with a variety of acquired disorders, ${ }^{1}$ in whom there would be no reason to suspect an abnormal pyramidal pathway projection does suggest that an abnormal corticomotoneuronal projection is not a necessary requirement for the appearance of mirror movements. This is supported by the normal projection found in our patient with acquired mirror movements as a result of internal capsular infarction. Where the abnormality lies in these patients is uncertain.

\section{Erratum}

Throughout the text (and in the table) the word "anticlockwise" should be replaced by "clockwise" and vice versa. Previous should be replaced by "clockwise" and vice versa. Previous may also contain this error (see JNNP 1990;53:707).

This study was supported by the DFG and EC. TCB is an EC Research Fellow.

1 Zülch KJ, Müller N. Associated movements in man. In Vinken PJ, Bruyn GW, eds. Handbook of clinical neurology, Vol 1. Amsterdam: North Holland, 1969: neurology

2 Myrianthopoulos NC. Mirror movements. In: Vinken PJ, Bruyn GW, eds. Handbook of clinical neurology, Vol 42 . Amsterdam: North Holland, 1982:233-234.

3 Avery LN, Rentfro CC. The Klippel-Feil syndrome: a pathologic report. Arch Neurol Psychiatr 1936;36: 1068-76.

4 Gunderson $\mathrm{CH}$, Solitare GB. Mirror movements in patients with the Klippel-Feil syndrome. Arch Neurol 1968; 18:675-9.

5 Conrad B, Kriebel J, Hetzel WD. Hereditary bimanual synkinesis combined with hypogonadotropic hypogonadism and anosmia in four brothers. I Neurol 1978;218:263-74.

6 Cohen LG, Bandinelli S, Lelli S, Hallet M. Non invasive mapping of hand motor somatotopic representation using magnetic stimulation. J Clin Neurophysiol 1988;5:371.

7 Farmer SF, Ingram DA, Roche SW, Stephens JA. Evidence that mirror movements in the Klippel-Feil syndrome in man are produced by activity in branched-stem presynaptic inputs from corticospinal neurones. $J$ Physiol 1989;418:102.

8 Barker AT, Jalinous R, Freeston IL. Non-invasive magnetic stimulation of human motor cortex. Lancet 1985;i:1 106-7.

9 Claus D, Flügel D, Brenner PM, Gropp N. Zum Einfluss der Parameter magneto-elektrischer Reize aud deren Wirkung im Nervensystem. $Z E E G E M G$ 1990;21:187.

$10 \mathrm{Hess}$ CW, Mills KR, Murray NMF. Responses in small hand muscles from magnetic stimulation of the human brain. J Physiol 1987;338:397-419.

11 Hess CW, Mills KR, Murray NMF, Schriefer TN. Magnetic brain stimulation: central motor conduction studies in multiple sclerosis. Ann Neurol 1987;22:744-52.

12 Ingram DA, Thompson AJ, Swash M. Central motor conduction in multiple sclerosis: evaluation of abnorconduction in multiple sclerosis: evaluation of abnormalities revealed by transcutaneous magnetic stimulation
of the brain. J Neurol Neurosurg Psychiatry 1988;51: of the bra 94 .

13 Forget R, Boghen D, Attig E, Lamarre Y. Electromyographic studies of congenital mirror movements. Neurology 1986;36:1316-22.

14 Kristeva R, Keller E, Deecke L, Kornhuber HH. Cerebral potentials preceding unilateral and simultaneous bilateral finger movements. Electroencephalogr Clin Neurophysiol 1979;47:229-38.

15 Roland PE. Organisation of motor control by the normal human brain. Human Neurobiol 1984;2:205-16. 